\title{
Mars Atmospheric Oxidant Sensor (MAOS): An In-Situ Heterogeneous Chemistry Analysis
}

\author{
A.P. Zent ${ }^{3}$ \\ R. C. Quinn ${ }^{b}$
}

F. J. Grunthaner ${ }^{c}$, M. H. Hecht ${ }^{c}$, M. G. Buehler ${ }^{\mathrm{c}}$

C. P. McKay

A. J. Ricco ${ }^{\text {d }}$

${ }^{a}$ MS 245-3

NASA Ames Research Center

Moffett Field CA, 94035 USA

Vox: $650-604-5517$

Fax: 650-604-6779

azent@mail.arc.nasa.gov

Correpsonding Author

b(SETI Institute) Ames Research Center

Moffett Field CA, 94035 USA

'Jet Propulsion Laboratory

Pasadena CA 91109 USA

${ }^{d A C L A R A}$ Biosciences

Mountain View CA 94043 USA.

Submitted to:

Planetary and Space Science 


\section{Abstract}

We describe a chemometric array sensor, the Mars Atmospheric Oxidant Sensor (MAOS, pronounced "mouse") that is designed measure the oxidation rate of thin films on the martian surface. We select films that are sensitive to particular types of oxidants, that represent key elements in the martian soil, or that emulate prebiotic materials. Concern that naturally arising martian oxidants may have destroyed evidence of ancient life on Mars was raised by the Viking mission in the 1970 's. The possibility that oxidants may limit the viability of biological habitats is particularly timely in light of recent suggestions of contemporary flowing water on Mars. By controlling the temperature of the films, as well as their exposure to dust and ultraviolet light, MAOS will discriminate among leading hypotheses for oxidant production. MAOS weighs 55 grams, fits in a $6 \times 7 \times 2 \mathrm{~cm}$ envelope, and uses $250 \mathrm{~mW}$ power. Much of the enabling technology was developed for the MOx experiment, lost on the Russian Mars ' 96 mission. 


\section{Introduction}

The planetary community is interested in life on other planets, as well as expanding the human presence into the solar system. On Mars, naturally arising oxidants may decompose organics relict of Mars' early history, and may affect human health and safety. In order to improve our chances of finding chemical evidence of life on Mars, and designing habitats and equipment that will function well on Mars' surface, we need to improve our understanding of these oxidants. We have developed, and describe here, a chemometric sensor array that we hope to use to characterize the oxidation potential of the Martian environment.

A quarter of a century ago, two Viking Landers were sent to Mars to seek evidence of life. However, the Viking experiments detected neither life nor organics in the martian soil. Vigorous chemical activity was interpreted as the action of oxidizing species in the soil, rather than as biology. Viking was not designed to study oxidants. It is not possible to deduce from those results the nature and prevalence of the oxidizing species, nor whether they originated in the atmosphere or the bulk soil itself. Even the relationship between the absence of organics and the presence of oxidants may be considered circumstantial

Our chemiresistor-based oxidation potential sensor is referred to here as the Mars Atmospheric Oxidant Sensor (MAOS, pronounced "mouse"). It weighs 55 grams, fits in a $6 \times 7 \times 2 \mathrm{~cm}$ box, and uses, in normal operation, $250 \mathrm{~mW}$ power. The fundamental unit of MAOS is the "chemical pixel," (Fig. 1) a $4 \times 2$ array of concentric electrodes coated with a single chemical film. Half of the electrodes are permanently sealed from the environment, and serve as references. With the 4 sensing electrodes, we examine the origins of Mars' oxidizing potential by monitoring the resistivity of the films as a function of time. We use two kinds of passive filters, one to exclude UV, and another to exclude dust. These two filters are deployed over the 4 sensing electrodes so that they exclude, a) UV, b) dust, c) both, and d) neither.

By monitoring differences in film reaction among the 4 electrodes, we will quantify the relative importance of soil-borne oxidants, UV photoxidation, and gaseous oxidants. Six identical chemical pixels are grouped into a "module". The total MAOS experiment consists of 8 modules, each featuring a unique chemical film. The thin film reactants include highly electropositive metals, semiconductors, and a set of organic functional groups. In an "experiment", we simultaneously expose one pixel from each module, and record the relative resistance of the 8 chemicals, in 4 filter configurations, (total of 32 channels of data) as a function of time. Experiments differ in the time of day at which they are initiated, in the thermal profile we impose on the active pixels, and in the differences in ambient dust and UV fluxes. MAOS can conduct 6 experiments. The final interpretive identification of oxidants will be made, if possible, in terrestrial laboratories by matching the reactivity pattern of these reference reactants with a series of synthesized oxidants. 


\section{Scientific Goals and Objectives}

\subsection{The Possibility of Martian Life, Past or Present}

There is ample evidence that Mars was once much warmer and wetter than it is today (e.g. Carr, 1996). Although the martian surface has not yet been dated, warm wet conditions may date from the period of pre-biotic chemical evolution and abiotic development of life on Earth $(3.85 \mathrm{Ga})$ (Schidlowski, 1988; Mojzsis, 1996). If martian life also arose in that early period, it is plausible that evidence persists at depth within the regolith (e.g. McKay, 1997).

Recent discoveries have emphasized the urgency of the search. The controversy surrounding Martian meteorite ALH84001 (D. McKay et al., 1996) broadly increased interest in martian life. Evidence of apparently recent water flow on the martian surface has further fueled speculation about geologically contemporary habitable environments (Malin and Edgett, 2000).

Our understanding of the potential habitability of Mars has been broadened by discoveries of extraordinarily tenacious terrestrial life. Organisms have been found at depths of $2 \mathrm{~km}$ in the Columbia River basalt (Stevens and McKinly, 1995), evidently directly metabolizing the igneous rocks in which they live. Karl et al. (1999) recently recovered, from ice at $4 \mathrm{~km}$ depth, just above the surface of Lake Vostok, Antarctica, samples with viable bacterial cells, suggesting that Lake Vostok contains viable microorganisms. Anaerobic organisms recovered from a depth of $2.7 \mathrm{~km}$ beneath Virginia live at temperatures of $75^{\circ} \mathrm{C}$. The microbes may have been trapped for 80 million years (Tseng et al., 1996). The limits of life do not rule out the persistence of life in sequestered habitats within the present-day martian regolith.

\subsection{The Viking Biology Results}

In response to telescopic observations through the 1950's suggesting that Mars could be the abode of life (e.g. Wyatt, 1964), the Viking mission was designed principally to search for such life. The Viking Gas Chromatograph Mass. Spectrometer (GCMS) indicated no organic compounds containing more than two carbon atoms at concentrations greater than parts per billion. Nor did it find any one- and two-carbon compounds at the parts per million level (Biemann et al., 1977, Biemann and Lavoie, 1979). This sensitivity level has recently been challenged (Benner et al., 2000; Glaven et al., 2001).

The absence, or in any event the depletion, of organics on Mars is a puzzle because, even in the absence of an in situ source, meteoritic infall will carry organics to Mars (e. g. Flynn and McKay, 1996). These and other results led to the hypothesis that the Martian surface material contains one or more oxidants that actively attack organic compounds (Klein 1978; 1979).

The Viking Biology experiments also provided evidence of soil components in a peculiar oxidation state. In the Gas Exchange experiment (GEx), exposure of soil to $\mathrm{H}_{2} \mathrm{O}$ vapor led to rapid release of $\mathrm{O}_{2}$, far in excess of the amount that could be produced by desorption (Oyama and Berdahl, 1977).

In the Labeled Release Experiment (LR), incubation of radioactively-labeled nutrient solution with Martian soil led to release of radioactively labeled $\mathrm{CO}_{2}$, presumably due to oxidation of the organics (Levin and Straat, 1977). However, because the LR activity was removed by heating, but the GEx activity was not, the oxidants responsible for them are believed to be different. 
It is generally held, but not demonstrably true, that the oxidant responsible for the GEx and LR results are also responsible for actively destroying incoming organics at the martian surface. In addition, these oxidants may have destroyed relatively refractory reduced compounds relict of pre-biological chemical evolution. These hypotheses can only be tested by identifying the origin and nature of the martian oxidants.

If oxidants render the near-surface Martian regolith sterile, then martian life must be sought in special subsurface environments (e.g. Boston et al., 1992). Unfortunately, the depth of the organic-free, apparently oxidizing, soil is unknown. Theoretical considerations however suggest that the likely depth of the oxidized layer, assuming little aeolian mixing, is on the order of $\mathrm{cm}$ to a few m (Bullock et al., 1994; Zent, 1998).

Sample return may not elucidate the oxidant question because it is clear that the martian oxidants break down spontaneously, because they are likely to be composed of common elements, and because the conditions required for their preservation are unknown (Zent and McKay, 1994).

\subsection{Nature of the martian oxidants}

A successful oxidant model must explain the release of $\mathrm{O}_{2}$ in the Viking Gas exchange experiment (GEx), the decomposition of added nutrient in the labeled release (LR), and the absence of organics in the GCMS.

The concentration of oxidants in the soil sufficient to account for the GEx and LR results is shown in Table 1. Zent and McKay (1994) have argued that the amount of oxidant required would cover less than $1 \%$ of the surface area of grains that compose Martian soil (Ballou et al., 1978). The soil concentration may be as low as a few ppm, which would not pose a serious hazard to humans or materials.

The nature of the oxidant has been discussed for over 20 years. (Figure 2). It has been argued by some that the oxidant must originate in the atmosphere, and hence may be able to diffuse through the regolith to unknown depths, wiping out the early chemical record as it goes (Hunten 1974). Others maintain that the oxidant is strongly bound to dust surfaces, and hence potentially hostile to humans and their habitats (Nussinov et al., 1988; Yen et al., 2000).

Oxidants do not intrinsically preclude the presence of organic material. SNC meteorites are also oxidizing (Gooding, 1992). There have, however, been reports of organic material in the SNCs (Wright et al., 1989), as well as in ALH84001 (D. McKay et al., 1996). It is also possible that the oxidizing species are virtually immobile, and could co-exist with organics (Zent, 2001).

Models of oxidant generation fall into several categories (Zent and McKay, 1994):

- Solar ultraviolet radiation may cause photolysis of atmospheric water vapor into "odd- $\mathrm{H}^{\prime}$ ( $\mathrm{H}$, $\mathrm{OH}, \mathrm{HO}_{2}$, and $\mathrm{H}_{2} \mathrm{O}_{2}$ ) (e.g. Hunten, 1974, Barth et al. 1992). Subsequent recombination may produce oxidizing species that precipitate on to the surface. The total oxidant load detected by Viking could have been produced in as little as 2-10 years by this mechanism (Kong and McElroy, 1977). Photochemical oxidant production ceases at sunset, and it has been hypothesized that some oxidants, such as hydrogen peroxide, which has roughly the same condensation properties as water, may precipitate at an accelerated rate just after sunset (Barth et al. 1992). Certianly $\mathrm{H}_{2} \mathrm{O}_{2}$ is capable of degrading Mars analog organics (McDonald et al., 1998). 
- Odd- $\mathrm{H}$ and odd-O groups produced by photolysis may subsequently complex with soils (e.g. Oro et al., 1976). In particular, transition metals are capable of forming complexes with peroxy radicals that behave much as the Viking biology samples did (Quinn and Zent, 1999). If a significant portion of the oxidative power is bound to soil surfaces, we will note a lack of reactivity behind our dust-filtered electrodes.

- UV-silicate interactions may lead to generation of physically trapped oxygen (Nussinov et al 1978) or radical species (Yen et al., 2000) directly in the silicate matrices. The non-bridging oxygen defects resulting from broken $\mathrm{Si}-\mathrm{O}$ bonds are mobile, and could in principal migrate through silicate lattices. The soil and dust surfaces would be strongly oxidizing, but the atmosphere itself need not be oxidizing. Mineral and organic analog films exposed to UV will quantify these reaction mechanisms most acutely.

- Dust clouds and frictional generation of glow discharges have been invoked (Mills, 1977), which would argue that the regolith is organic- free to whatever depth is scoured by wind..

- Other mechanisms require both UV and atmospheric oxidants. The dangling bonds from radiation damage are highly reactive, and could easily form semi-permanent complexes, such as such as perchlorates from photolyzed, complexed halide compounds (Zent and McKay, 1994).

- There may be bulk phase superoxidants of unknown origin (e.g. Ponnamperuma et al., 1977; Ballou et al., 1978; Tsaipan et al., 1999).

- Banin and Rishpon, (1979) and Banin and Margulies (1983) argued that the gas released in the LR experiment was due to intrinsically reactive clays, not oxidants. This hypothesis demands a separate mechanism to explain the lack of organics.' Stoker and Bullock (1997) argue that the UV flux at the martian surface is adequate to explain the lack of organics.

\subsection{MAOS' Scientific Objectives}

In order to explore the relative importance of these hypothetical mechnisms, MAOS will record, as a function of temperature, the differential DC resistivity of each element of an array of 192 active films relative to an identical sealed reference film. A subset of the films will be exposed to sunlight, a second to dust, a third to the combination of dust and sunlight, and a fourth only to the air.

MAOS operations are divided into six experiments, each of which involves exposure of 8 types of chemical films to the martian environment. The experiments differ only in the environmental conditions and the applied temperature profiles. During any experiment, each of the eight films is deployed in four configurations (sharing a common heater) to separate the effects of air, dust, and ultraviolet illumination. Thus, for any experiment, the returned data consists of 32 resistance values relative to 32 sealed reference resistors, and 8 temperature readings.

Film resistivity is the dependent variable in the MAOS experiment. It is measured with respect to the independent variables of temperature, UV flux, and dust and soil loading. To control and record the temperature of each of the $4 \times 2$ pixels, a resistive ruthenium oxide heater is patterned on the substrate. The high thermal conductivity of the components will ensure uniform temperature across the pixel: Temperature is monitored with a small 12-bit digital serial thermometer on the sapphire surface.

The experimental protocol calls for sampling the integrating films over an extended period (up to a martian year, approximately 2 Earth years). In specific campaigns the temperature of the films 
can be raised to increase the chemical activity of adsorbed molecules, and thermal acceleration of very slow reactions.

\section{Hardware Implementation}

\subsection{Operating principles}

In MAOS, the DC resistance of a thin solid-state film, deposited by evaporative deposition or solvent casting over a set of noble metal electrodes, is monitored. The electrode gap and spatial configuration are chosen to maximize the sensitivity of the thin film to oxidation. The resistance is an extremely sensitive function of the ambient gases. There are a variety of chemical mechanisms that can alter the conductivity of the films.

At the lower thickness, $(10-20 \mathrm{~nm})$ thin metal films consist of closely spaced clusters or islands with very large surface to volume ratios. At higher thickness, the films become continuous, but the surface contribution is still substantial. The measured resistivity of such thin films has a surface and a bulk component. With oxidation, a surface oxide is formed, severely changing carrier transport at the Fermi surface. Oxidation also results in conversion of some metal to nonconducting oxide, thereby reducing the cross-section for metallic conduction and increasing the effective resistivity of the film. In the limit, the film is completely oxidized, becoming an insulator. The extent of reaction is monitored by following the resistivity or impedance of the chemiresistor.

Semiconductor films, as a group, tend to be sensitive to reduction/oxidation reactions, because conductivity changes are related to changes in the carrier (hole) concentrations across their surfaces. In the case of an oxidizing environment such as Mars, a change in carrier concentration occurs when electrophilic gases abstract electrons from the conduction band, and become immobilized on the surface. The equilibrium conductivity varies with the type and abundance of oxidant because of differences in equilibrium adsorptive coverage and differences in the mobility of the sorbed ions (Gardener et al., 1992).

The observed resistance is also affected by temperature, surface morphology, and the details of the contact of the thin film to the noble metal of the electrode array. Because of the relative precision and accuracy with which current and voltage can be determined, the chemiresistor is one of the best transducer configurations for high sensitivity applications. A further advantage of this implementation is that chemiresistors can be actively probed or read during storage, as well as during transit.

\subsection{Instrumentation}

The MAOS experiment places an array of well-characterized thin films in intimate contact with the martian atmosphere, exposing it to dust and UV (individually and in combination) while controlling the temperature of the contacting interface.

MAOS is shown schematically in Figure 3. The reaction sensor arrays are made of a chemically inert substrate, filter sets for isolating the effects of UV and dust on chemical reactivity, and silicon nitride hermetic seals for maintaining film integrity prior to deployment. MAOS detection sensitivity ranges from a few tenths of a monolayer to several monolayers of reaction product. 
The $60 \times 70 \times 20 \mathrm{~mm}$. package has the sensor array on the front surface The sensor array is directly mounted to the electronics support board that contains the measurement circuitry.

\subsubsection{Sensing films}

MAOS chemiresistor transducers are thin films deposited on highly insulating substrates. The resistivity of each thin film is measured to determine the extent of film oxidation (Fig. 4).

The active chemical sensing films are deposited through a contact shadow mask such that the deposited area covers the gold contacting electrodes with a generous margin. For deposition control, no less than 20 substrates are deposited simultaneously. The resistivity of several substrates is monitored during the deposition in order to keep the final film thickness within acceptable margins.

Eight film types and several backup choices were selected from a candidate list of 25 .

Silver $(15 \mathrm{~nm})$ : Extremely reactive to oxidants, particularly $\mathrm{O} \cdot$ and $\mathrm{O}_{3}$.

Gold $(10 \mathrm{~nm})$ : Relatively inactive with respect to oxidants, but (reversibly) sensitive to frost or thin water films.

Iron $(25 \mathrm{~nm})$ : A transition metal with complex oxidation chemistry. Several species can form, depending on the strength of the oxidant. This film models the response of the iron-rich soil to oxidants.

Alkane thiol ( 1 monolayer) on gold $(10 \mathrm{~nm}$ ). Thiol molecules form a self-assembled monolayer on the gold base, strongly modifying the gold conductivity. Oxidation restores the gold conductivity to its original state. This fully aliphatic long chain thiol mimics meteoritic kerogen, the surface of cell walls, and chemical degradation products from biogenic processes.

d-Cysteine or l-Cycsteine (1 monolayer) on $A u(10 \mathrm{~nm})$ : An amino acid similarly bonded to a thiol group, forming a self-assembled monolayer on the Au base. Cysteine/Au is sensitive to organic group oxidation, and therefore effectively monitors contaminants.

$C_{60}(120 \mathrm{~nm})$ : A carbonaceous material, sensitive to combinations of $U V$ and oxidants.

Lead sulfide $(100 \mathrm{~nm})$ : A semiconductor that is very reactive to oxidants and which exhibits a strong response to peroxide and oxide radicals. Semiconducting films show the strongest response to oxidation in our laboratory experiments because the fundamental charge transport mechanism within the film can change with oxidation.

Copper pthalocyanine $(250 \mathrm{~nm})$ : A well-characterized, traditional chemical sensor for oxidants, useful over a large dynamic range. (e.g. Wohltjen et al., 1985).

\subsubsection{Seals}

The only sensor deployment system used by MAOS is the electrically-triggered rupturing of thin silicon nitride membrane seals, individually addressable on each chemical pixel.

The effectiveness and reproducibility of the MAOS reference reactants depends on the extent to which they are delivered in pristine condition to their destination. To accomplish this, the films are encapsulated in a hermetically sealed enclosure. A micromachined top seal cover is bonded to the substrate immediately following sensor film deposition. The seal is strong enough to withstand more than 15 psi gauge differential across the membrane and has been tested to vibration 
loads of more than $500 \mathrm{G}$ using the Proton launch vibration spectrum. (Manning et al., 1997). On computer command, a seal is deployed by rapidly heating. The sudden temperature increase thermally stresses the film, which decomposes into micron sized particles, exposing the chemical pixel. These particles are chemically inert, and will have negligible impact on the experiment.

\subsubsection{Filters}

Captured within the silicon frame and suspended above the sensors are porous polymer dustfilters, transparent to the ultraviolet and permeable to atmospheric gases. The dust filter will cover two of the four exposed sensors in each chemical pixel. External to the silicon nitride seal is an awning that blocks two of the sensors (one of which has a dust filter) from ultraviolet light.

\subsection{Performance characteristics}

MAOS sensors will respond to a variety of stimuli that will be distinguished using pattern recognition techniques.

Figs.4a through $4 \mathrm{~g}$ display representative responses for many of the MAOS films. All of these data were taken at $9.3 \mathrm{mbar}$ in a Mars-like atmosphere. Figs. $4 \mathrm{a}$ and $4 \mathrm{~b}$ show the response of two self-assembled monolayers (deposited on thin gold films) to the a pulse of $40 \mathrm{ppm}$ ozone. Both show a reduction in resistance, but the magnitude and rate of change are quite different for the amino acid (cysteine) and the simple hydrocarbon. The response of the CuPc chemiresistor (Fig. $4 \mathrm{c}$ ) to $20 \mathrm{ppb}$ of $\mathrm{O}_{3}$ shows a change of more than $10 \mathrm{M} \Omega$. The signal is sufficiently strong that $10^{9} \mathrm{~cm}^{-3}$ ozone molecules can readily be detected.

Figs. $4 \mathrm{~d}$ and $4 \mathrm{e}$ show the response of iron and fullerene thin films to $50 \mathrm{ppb}$ of anhydrous hydrogen peroxide generated by decomposition of the peroxide-urea complex. The response of the iron sensor is moderate and integrating, while the $\mathrm{C}_{60}$ film changes drastically. On Mars, reactions in bulk soil may show different response during nighttime heating of the films, when adsorbed $\mathrm{H}_{2} \mathrm{O}$ from the saturated boundary layer may activate the soil oxidants. In Fig. 4f, $200 \mathrm{ppm} \mathrm{KO}_{2}$ has been mixed with Mauna Kea palagonite. The resistance of a $150 \AA \mathrm{Ag}$ sensing film is seen to be insensitive to the presence of the dry, solid $\mathrm{KO}_{2}$. No reaction takes place until the abundance of $\mathrm{H}_{2} \mathrm{O}$ reaches approximately 5 parts per thousand in the laboratory air.

Fig. $4 \mathrm{~g}$ shows the response to condensation of water on a silica substrate. Resistance is measured over a $15 \mu \mathrm{m}$ gap. As the temperature on the substrate is lowered, the resistance drops markedly at first condensation (the dewpoint is $-19^{\circ} \mathrm{C}$ ).

\subsection{Analysis}

Interpretation of the MAOS data requires careful characterization of the films and the contextual environment, both on Earth and on Mars. Pattern recognition techniques will enhance quantitative analysis.

The primary MAOS objective, to measure the relative effect of air, dust, temperature, and ultraviolet light on reaction rates, will not require elaborate pattern recognition techniques. Nor will the second MAOS objective, to determine the oxidation potential and rate of oxidation. These rely on careful laboratory simulation and analysis. Beyond these objectives, however, the power of chemometric arrays lies in their combination with pattern recognition techniques. 
Chemical microsensors do not provide selective responses to specific molecules. Rather, selectivity is obtained through computational techniques that compare the response of the array to unknown analytes against a reference set of responses previously obtained from known analytes. Multivariate techniques are needed both in the array design phase and during array use in the field.

\subsection{Calibration}

MAOS sensors will be characterized with reference resistors on Mars, and against sophisticated analytical instruments on Earth.

The calibration program begins with surface characterization at the time of sensor film deposition. X-ray. Photoelectron Spectroscopy will be used to look at the quality of film and coverage, and to evaluate contamination from the deposition system. These same techniques will be employed on the deposition surfaces, before and after deposition, to verify the cleanliness of the surfaces. Ellipsometry will be employed to measure film thickness, and atomic force microscopy (AFM) to assess morphology.

Laboratory models will be subject to extensive laboratory calibration of resistance as a function of oxidation, temperature, and illumination. Identical to the flight sensors, these test articles will be opened under Mars conditions. Resistivity will monitored relative to a reference electrode in the presence of UV light, dust, and various gas phase and interfacial oxidants.

Once on Mars, high performance reference resistors will be used to calibrate the electronics, and to monitor possible degradation of films during storage launch, and cruise.

Finally, witness sensors, identical to the flight units, will be maintained on Earth in environmental conditions similar in temperature and pressure to those experienced by the flight films. These units will be used to assess any long-term changes in sensor response.

\subsection{Interferences}

To ameliorate most interference, MAOS employs a four-fold passive filtering approach that separates the effects of dust and sunlight on oxidation.

Possible interference in the resistivity signal are due to environmental processes that foster additional charge separation. Solar illumination of the fully-exposed films may produce transient carrier excitation, thereby changing bulk resistance. There may also be photoelectrons, photocurrents or photovoltages affecting measurements. Dust can acquire substantial surface charge in the low humidity environment, and may be responsible for charging film surfaces. Wind, if velocities are high enough, may result in triboelectric charging.

To accomplish the required shielding from UV irradiation for one half of all exposed sensors, an awning will be placed $2 \mathrm{~mm}$. above the surface of the sensors. In order to discriminate chemical effects caused by the presence of accumulated dust grains, half of the exposed sensors are covered with a gas-permeable membrane approximately 15 microns thick, with a pore size distribution centered at 1.2 microns in diameter. This filter is placed within the silicon-based hermetic seal assembly and bonded in place. With no strong gas flow, no blockage of the membrane is anticipated from particles down to 0.1 microns in diameter. The fluoropolymer has a UV cutoff near $200 \mathrm{~nm}$, and will therefore transmit all but a minor portion of the UV illumination spectrum. 
System outgassing is viewed as the only potential source of actual chemical contamination of the system. Outgassing is more likely to be a problem during cruise, when MAOS is safely sealed, and degassing should be nearly complete by EDL.

\section{Summary}

In order to take definitive steps toward identifying the nature and origin of oxidants in the martian surface materials, to guide future astobiological exploration of Mars, and to assist in design of equipment and procedures for future human exploration, we have developed the MAOS instrument. MAOS is a chemometric, chemiresistor-based sensor array that will measure the thin film reaction rates of a variety of materials as a function of dust abundance, UV flux, and temperature. Analysis of MAOS data will allow us to quantify the in situ organic oxidation rate, as well as the overall oxidation potential. Utilized with sophisticated pattern recognition software, analysis of the MAOS data should allow us to determine the functional groups responsible for the oxidation potential of the martian dust and air. 


\section{References}

Ballou E. V., P. C. Wood, T. Wydeven, M. E. Lehwalt, R. E. Mack, Chemical interpretation of the Viking 1 life detection experiment, Nature, 271, 644-645, 1978.

Banin, A., J. Rishpon, Smectite clays in Mars soil: Evidence for their presence and role in viking biology experimental results, J. Molec. Evol., 14, 133-152,1979

Banin A., L. Margulies, Simulation of Viking biology experiments suggests smectites not palagonite as martian soil analogs, Nature, 305, 523-526, 1983

Barth, C. A. A. I. F. Stewart, S. W. Bougher, D. M. Hunten, S. J. Bauer, A. F. Nagy, Aeronomy of the current martian atmosphere, In: Mars, University of Arizona Press, Tucson, 1054-1089, 1992

Benner, S. A., K. G. Devine, L. N. Matveeva, D. H. Powell, The missing organic molecules on Mars, Proc. Natl. Acad. Sci., USA, 97, 2425-2430, 2000.

Biemann, K., J. Oro, P. Toulmin III, L. E. Orgel, A. O. Nier, D. M. Anderson, P. G. Simmonds, D. Flory, A. V. Diaz, D. R. Ruchneck, J. E. Biller, A. L. LaFleur, The search for organic substances and inorganic volatile compounds in the surface of Mars, J. Geophys. Res., 82, 4641-4658.,1977.

Biemann, K., J. M. Lavoie, Some final conclusions and supporting experiments related to the search for organic compounds on the surface on Mars, J.Geophys. Res., 84, 8385-8390, 1979

Boston, P. J., M. V. Ivanov, C. P. McKay, On the possibility of chemosynthetic ecosystems in subsurface habitats on Mars, Icarus, 95, 300-308, 1992.

Bullock, M. A., C. R. Stoker, C. P. McKay, A. P. Zent, A coupled soil-atmosphere model of $\mathrm{H}_{2} \mathrm{O}_{2}$ on Mars, Icarus,107, 142-154, 1994.

Carr, M. H., Water on Mars, Oxford University Press, Oxford, 1996

Flynn, G. J., D. S. McKay, An assessment of the meteoritic contribution to the martian dust, J. Geophys. Res. 95, $14,497-14,509,1990$.

Gardener, J. W., M. Z. Iskandrarani, B. Bott, Effect of electrode geometry on gas sensitivity of lead phthalocyanine films, Sensors and Actuators, -B Chem., 9, 133-142, 1982.

Glavin, D. P., M. Schubert, O. Botta, G. Kminek, J. L. Bada, Detecting pyrolysis products from bacteria on Mars, Earth Planet. Sci. Lett., 185, 1-5, 2000

Gooding, J. L., Soil mineralogy and chemistry on mars: Possible clues form salts and clays in SNC meteorites, Icarus, 99,28-41, 1992

Hunten, D. M., Aeronomy of the lower atmosphere of Mars, Rev. Geophys. Space Phys. 12, 529-535, 1974

Kari, D. M., D. F. Bird, K. Björkman, T. Houlihan, R. Shackelford, L. tupas, Microorganisms in the accreted ice of Lake Vostok, Antarctica, Science, 286, 2144-2147, 1999

Klein, H. P., The Viking biological experiments on Mars, Icarus, 34, 666-674., 1978.

Klein, H. P., The Viking Mission and the search for life on Mars, Rev. Geophys. Space Phys., 17, 1655-1662, 1979.

Kong, T. Y., M. B. McElroy, Photochemistry of the martian atmosphere, Icarus, 32, 168-189, 1977

Levin, G. V., P. A. Straat, Recent results from the Viking labeled release experiment on Mars, J. Geophys. Res., 82, 4663-4668, 1977

Malin, M.C., and K.S. Edgett, Evidence for recent groundwater seepage and surface runoff on Mars, Science, 288, $2330-2335,2000$

McDonald, G. D., E. deVanssay, J. R. Buckley, Oxidation of organic macromolecules by hydrogn peroxoide: Implications for stability of biomarkers on Mars, Icarus, 132, 170-175, 1998.

McKay, C. P., The search for life on Mars, Origins of Life and Evol. Biosph. 27, 263-289, 1997 
McKay, D. S., E. K. Gibson, K. L. Thomas-Keprta, H. Vali, C. S. Romanek, S. J. Clemett, X.D. F. Chillier, C. R. Maechling, R. N. Zare, Search for past life on Mars: Possible relic biogenic activity in martian meteorite ALH84001, Science, 273, 924-930, 1996.

Mills, A. A., Dust cloud and frictional generation of glow discharges on Mars, Nature, 268, 614, 1977.

Nussinov, M. D., Y. B. Chernyak, J. L. Ettinger Model of the fine-grained component of martian soil based on Viking lander data, Nature, 274, 859-861, 1978.

Oró, J., G. Holzer, The photolytic degradation and oxidation of organic compounds under simulated martian conditions, J. Molec. Evol., 14, 153-160, 1979.

Oyama, V. I., B. J. Berdahl, The Viking gas exchange experiment results from Chryse and Utopia surface samples, J. Geophys. Res., 82, 4669-4676, 1977.

Ponnamperuma, C., A. Sahimoyama, M. Yamada, T. Hobo, R. Pal, Possible surface reactions on Mars: Implications for Viking Biiology results, Science, 197, 455-457, 1997

Quinn, R. C., A. P. Zent, Peroxide-modified titanium dioxode: A chemical analog for putative martian soil oxidants, Orig. Life Evol. Biosph. 29, 59-72, 1999.

Stevens, T.O., and J.P. McKinley, Lithoautotrophic microbial ecosystems in deep basalt aquifers. Science, 270:450452, 1995

Stoker, C. R., M. A. Bullock, Organic degradation under simulated martian conditions, J. Geophys. Res., 102, 10881-10888, 1997.

Tsapin, A. I. M. G. Goldfield, G. D. McDonald, K. H. Nealson, B. Moskovitz, P. Solheid, K. M. Kemner, S. D. Kelly, K. A. Orlandini, Iron(VI): Hypotheitcal candidate for the martian oxidant, Icarus 147, 68-78, 2000

Wohltjen et al., 1985

Wright, I. P., M. M. Grady, C. T. Pillinger, Organic materials ina martian meteorite, Nature, 340, 220-222, 1989

Wyatt, S. P., Principles of Astronomy, Allyn and Bacon, Boston, 1964.

Yen, A. S., S. S. Kim, M. H. Hecht, M. S. Frant, B. Murray, Evidence that the reacitvity of the martian soil is due to superoxide ions, Science, 289, 1909-1912, 2000.

Zent, A. P., C. P. McKay, The chemical reactivity of the martian soil and implications for future missions, Icarus, 108, 146-157, 1994

Zent, A. P., On the thickness of the oxidized layer of the martian regolith, J. Geophys. Res., 103, 31491-31498, 1998.

Zent, A. P., Strategic reevaluation of the search for martian organics, Submitted: Proceedings of SPIE, 4495, 2001., 


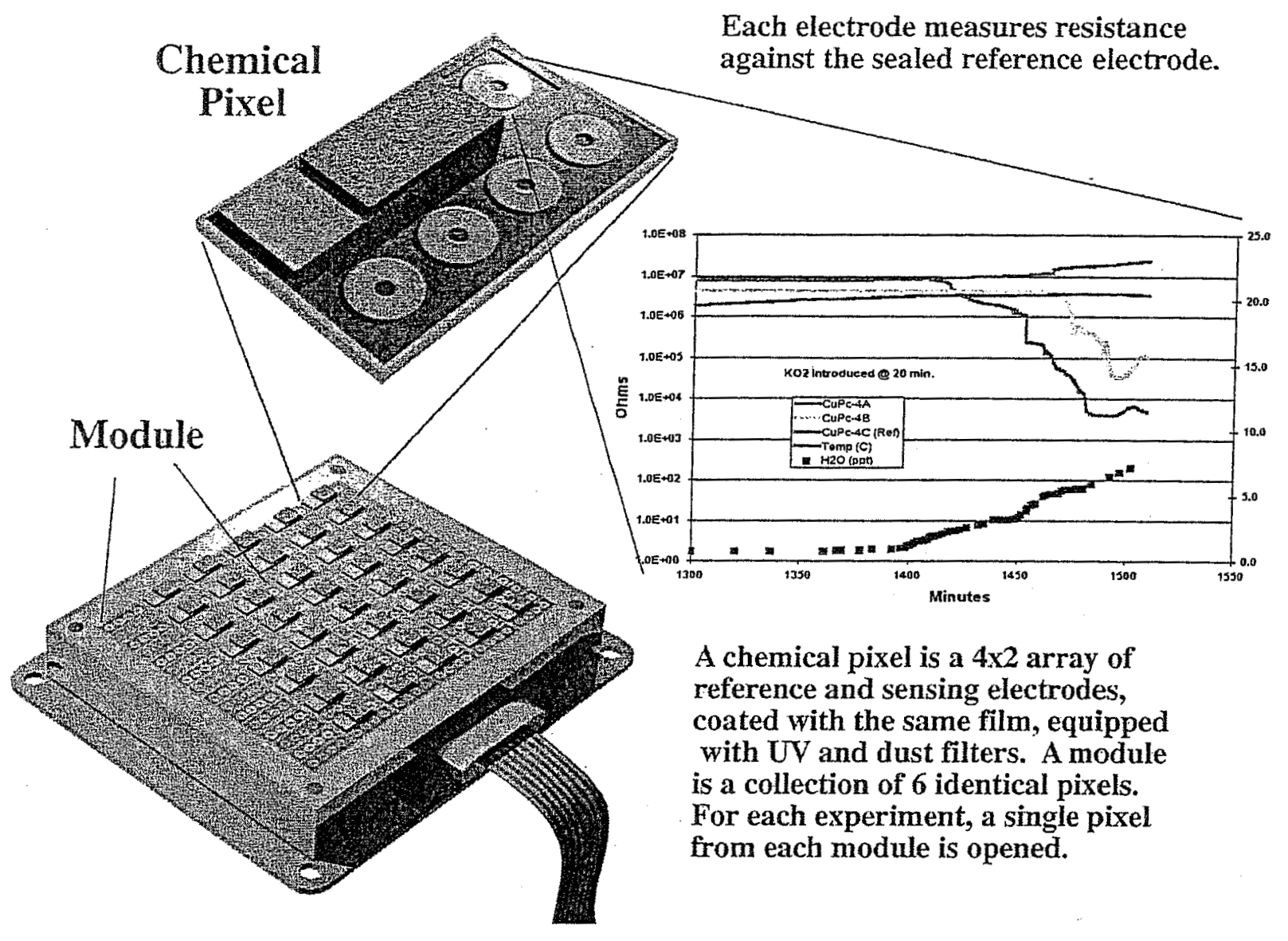

Figure 1. Cartoon of the MAOS Concept 


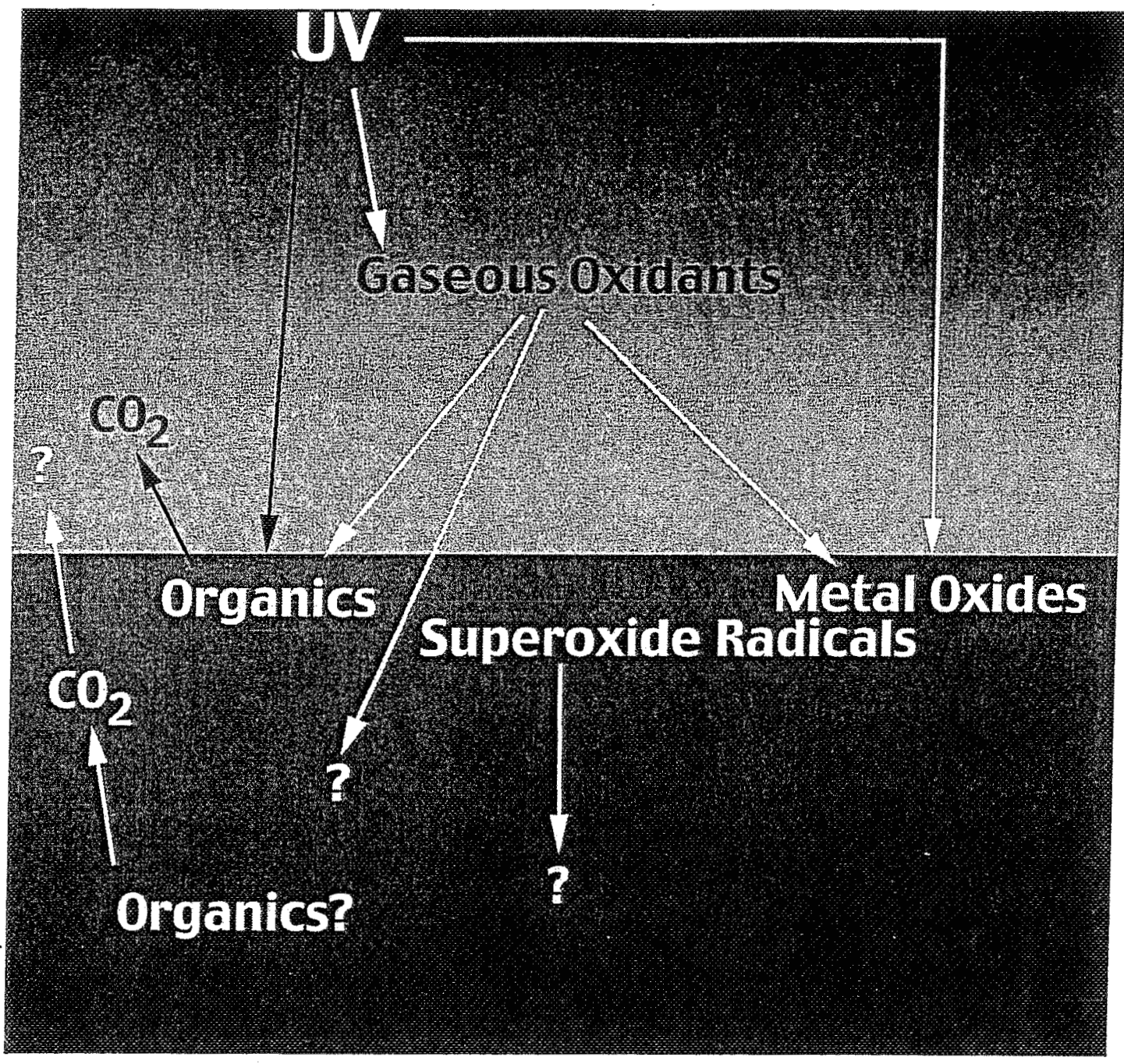

Figure 1.2 MAOS tests hypotheses of sources and sinks of martian oxidants 

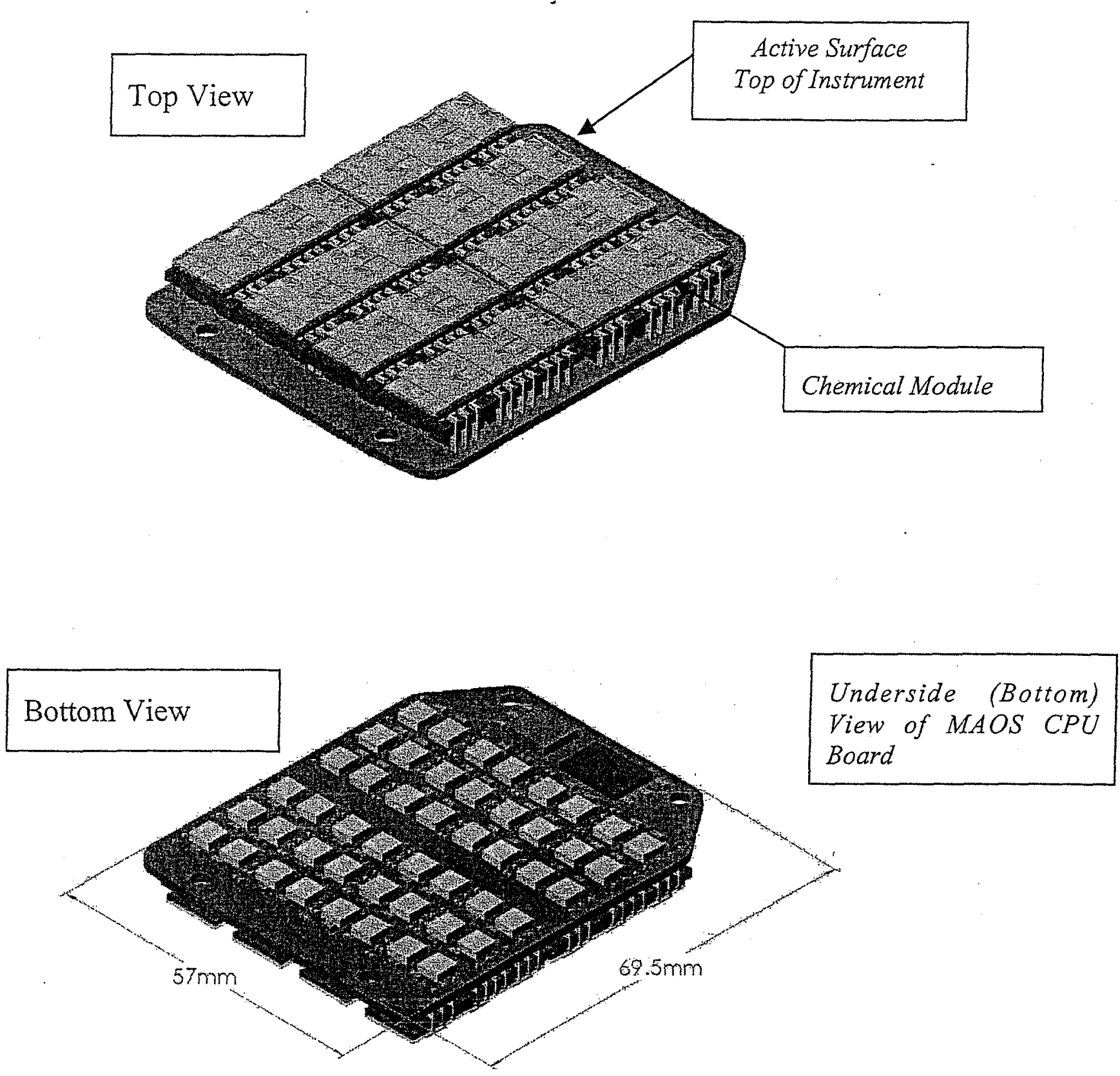

Fig. 3: Isometric drawing of full MAOS instrument with dimensions in mm. Total height from the top of the UV shade filter to the thickest component of the underside of the CPU board is $12.5 \mathrm{~mm}$. The upper overlay shows the eight different chemical modules with filters and seals in place. All component boards are populated with active components on both sides. The lower underlay shows the bottom side of the MAOS instrument CPU board with the switch array and PIC controller (A/D not shown). 


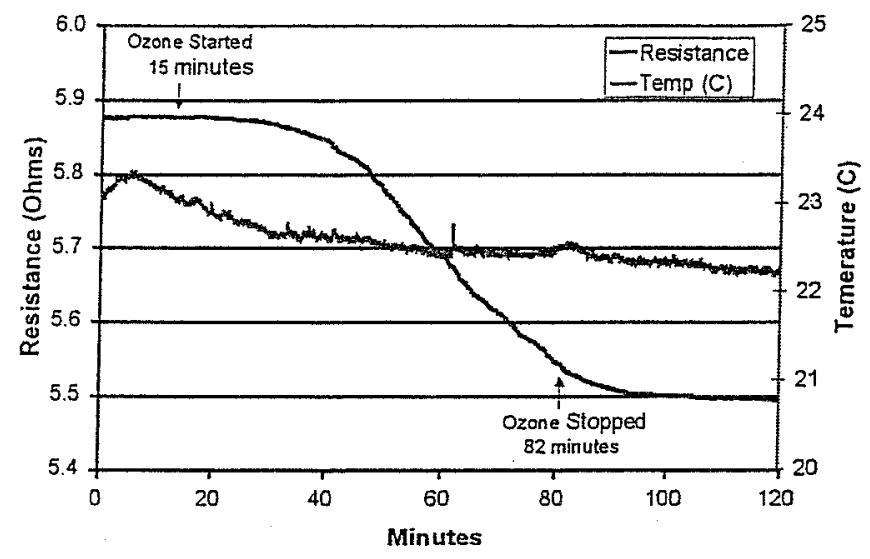

Fig 4a. Reaction of 1-Heptanethiol selfassembled monolayer bonded to a $40 \AA$ gold film with $40 \mathrm{ppb}$ ozone

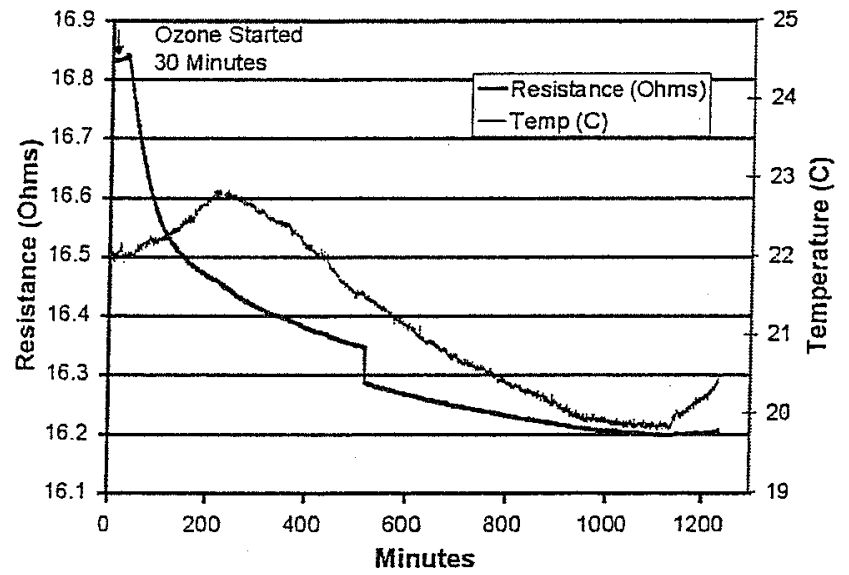

Fig 4b. Reaction of L-Cysteine self-assembled monolayer bonded to a thin 40 Angstrom gold film with $40 \mathrm{ppb}$ ozone.

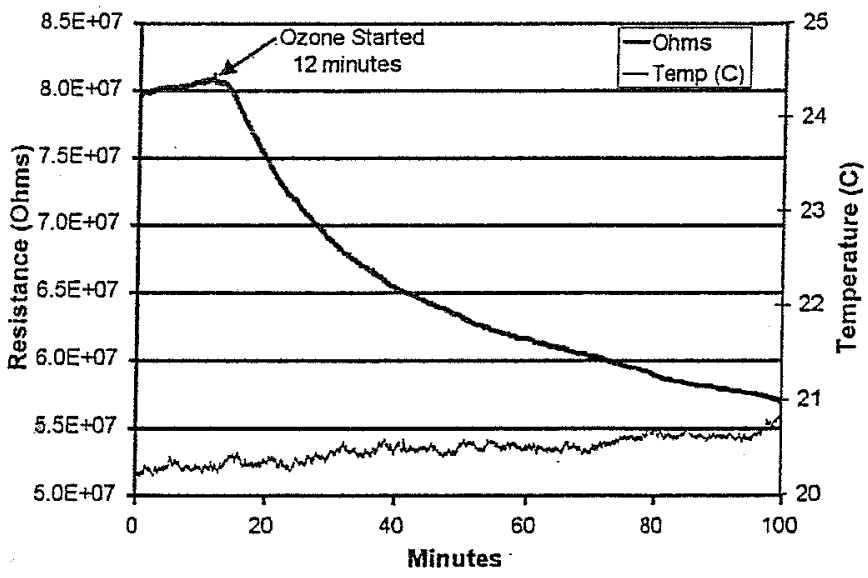

Fig 4c. Reaction of 1000-Angstrom-thick copper phthalocyanine film with $20 \mathrm{ppb}$ ozone. 


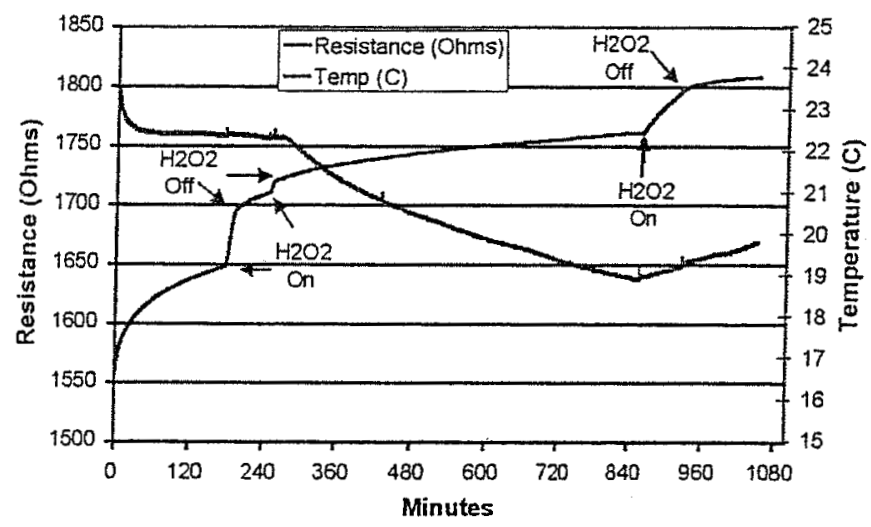

Fig. 4d. Reaction of a 100-Angstrom thick iron thin film with $50 \mathrm{ppb}$ anhydrous hydrogen peroxide vapor

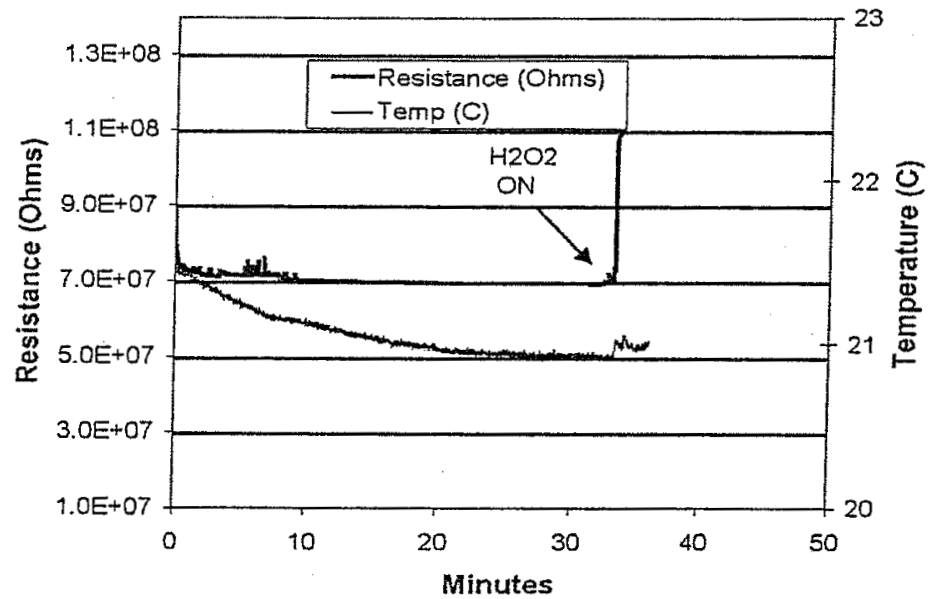

Fig. 4e. Reaction of a 2000-Angstrom C60 fullerene film with $50 \mathrm{ppb}$ anhydrous hydrogen peroxide vapor.

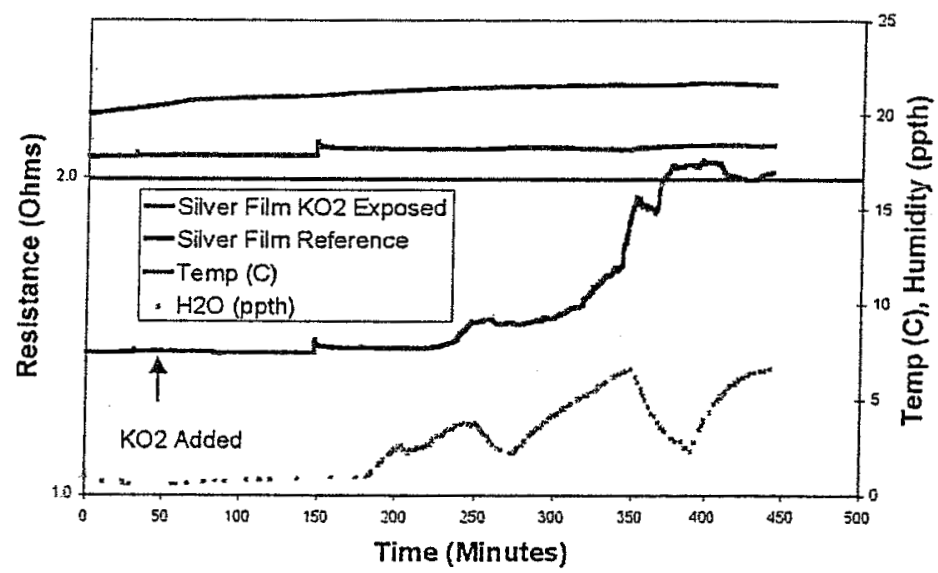

Fig. $4 \mathrm{f}$. Reaction of a 150- $\AA$ silver film with $200 \mathrm{ppm} \mathrm{KO}_{2}$ in palagonite. $\mathrm{Ag}$ reference exposed to palagonite without $\mathrm{KO}_{2}$.

Gas phase oxidant exposure was done with a flowing carrier gas at $90 \mathrm{sccm}$. The gas composition was $0.13 \%$, $2.7 \% \mathrm{~N}_{2}, 97.17 \% \mathrm{CO}_{2}$ and the chamber pressure was maintained at 9.3 mbar to simulate Mars conditions.

Thin semi-transparent films of gold, thin films of silver, iron, copper phthalocyanine and fullerene were deposited by vacuum evaporation. Self-assembled monolayers were deposited by immersing a 40 A thin gold films on silica substrates in a water/alcohol solution. 
Table 1. Comparison of $\mathrm{GEx} \mathrm{O}_{2}$ and $\mathrm{LR}^{14} \mathrm{C}$ results (After Klein, 1978).

\begin{tabular}{lllll}
\hline Sample & $\begin{array}{l}\mathrm{GEx} \mathrm{O}_{2} \\
\left(\mathrm{nmoles} / \mathrm{cm}^{3}\right)\end{array}$ & $\begin{array}{l}\text { Possible oxidant } \\
\left(\mathrm{ppm} ; \mathrm{KO}_{2} \rightarrow \mathrm{O}_{2}\right)\end{array}$ & $\begin{array}{l}\mathrm{LR} \mathrm{CO} \\
\left(\mathrm{nmoles} / \mathrm{cm}^{3}\right)\end{array}$ & $\begin{array}{l}\text { Possible oxidant } \\
\left(\mathrm{ppm} ; \mathrm{H}_{2} \mathrm{O}_{2} \rightarrow \mathrm{O}\right)\end{array}$ \\
\hline VL1 Surface & 770 & 35 & 30 & 1 \\
VL 2 Surface & 194 & 10 & 30 & 1 \\
\hline
\end{tabular}

